\title{
Etanercept (Enbrel): update on therapeutic use
}

\author{
George Spencer-Green
}

\begin{abstract}
Tumour necrosis factor (TNF) is an important inflammatory disease mediator in a wide spectrum of articular diseases, including adult and juvenile rheumatoid arthritis (RA, JRA). Etanercept (Enbrel), approved in the United States and in Europe for use in patients with RA and JRA, is an effective inhibitor of TNF that has been shown to provide rapid and sustained improvement in both of these diseases. Long term studies continue to show that etanercept controls signs and symptoms of RA and JRA with no change in rate or type of adverse event over time. To demonstrate that etanercept is effective as first line treatment for patients with early active $R A$ who have not been previously treated with methotrexate, and to examine the effect of etanercept on radiographic progression, a double blind, placebo controlled study was recently conducted, comparing etanercept with methotrexate (median dose 20 mg per week). Both etanercept $25 \mathrm{mg}$ twice weekly and rapidly escalated methotrexate were effective in reducing the signs and symptoms of RA, and etanercept was significantly better than methotrexate in slowing the rate of radiographic erosions. In patients with severe psoriatic arthritis (PsA), a double blind, placebo controlled study demonstrated that etanercept was also effective in reducing disease activity in PsA. Etanercept has been well tolerated in all of these clinical trials and offers an important new treatment option to patients with inflammatory articular diseases. (Ann Rheum Dis 2000;59(suppl I):i46-i49)
\end{abstract}

Tumour necrosis factor and etanercept The role of tumour necrosis factor (TNF) in rheumatoid arthritis (RA) and other rheumatological diseases has been previously described. ${ }^{1}$ Produced by synoviocytes and macrophages, TNF activates the endothelium, stimulates fibroblast proliferation, induces matrix metalloproteinase production, stimulates production of interleukin 1 (IL1) and interleukin 6, and activates osteoclasts via IL1 stimulation..$^{2-7}$

Recombinant human soluble TNF receptor (etanercept) is a dimeric fusion protein consisting of the extracellular portion of the human p75 TNFR linked to the Fc portion of a type 1 human immunoglobulin (IgG1). ${ }^{8}$ The primary action of etanercept is to bind and inactivate soluble and cell bound TNF and lymphotoxin $\alpha$. Although the $F c$ region contains the complement binding domain, etanercept does not fix complement or lyse TNF expressing cells in vitro. ${ }^{9}$ In addition, the presence of the Fc portion of human IgG1 in etanercept results in a relatively long median half life of 4.8 days and a high binding affinity $\left(\mathrm{K}_{\mathrm{i}}=10^{-10} \mathrm{M}\right){ }^{8}$ Etanercept is effective in suppressing inflammation in animal models of arthritis. ${ }^{10}$

\section{Etanercept clinical trials}

Positive results from phase I and II trials led to further evaluation of etanercept in patients with active RA whose disease was not controlled by disease modifying antirheumatic drugs (DMARDs). ${ }^{11}{ }^{12}$ Phase III studies have confirmed that patients show rapid and sustained improvement in adult and juvenile RA (JRA) disease activity and functional status during etanercept treatment. ${ }^{13-15}$ Etanercept has been approved in the United States and Europe for use alone or in combination with methotrexate in patients with RA and JRA who have failed treatment with at least one other DMARD. Etanercept was recently approved in the United States for use as first line treatment for reduction of signs and symptoms of disease and prevention of structural damage in patients with RA.

LONG TERM ETANERCEPT IN DMARD REFRACTORY RA PATIENTS

Patients from clinical trials of DMARD refractory RA ( $n=713$ ) were eligible to continue with etanercept treatment and have been followed up for up to 41 months (1152 patient years). ${ }^{16}$ Data from these patients show that etanercept has been well tolerated. There was no change in rate or type of any adverse event with prolonged use of etanercept, and the rate of infections associated with hospitalisation and/or use of intravenous antibiotics did not increase over time. No instances of opportunistic infection or tuberculosis have been seen. In all clinical trials of etanercept in DMARD refractory RA, the number of reported cases of cancer has been similar to the expected rate in the general age and sex matched population calculated from the National Cancer Institute's Surveillance, Epidemiology, and End Results (SEER) database. ${ }^{17}$ There has been no predominant cancer type. Overall, the types and rates of adverse events seen in open label extensions were similar to those seen in controlled studies of etanercept. Of the patients treated with 24 months of continuous etanercept treatment, $76 \%$ achieved at least an ACR20 response, $44 \%$ an ACR50, and $26 \%$ an ACR70. Of the 388 patients who were receiving baseline corticosteroid treatment in these studies, $17 \%$ were able to discontinue corticosteroid use, $54 \%$ were able to decrease their doses, and only $7 \%$ required a dose increase. ${ }^{16}$ 


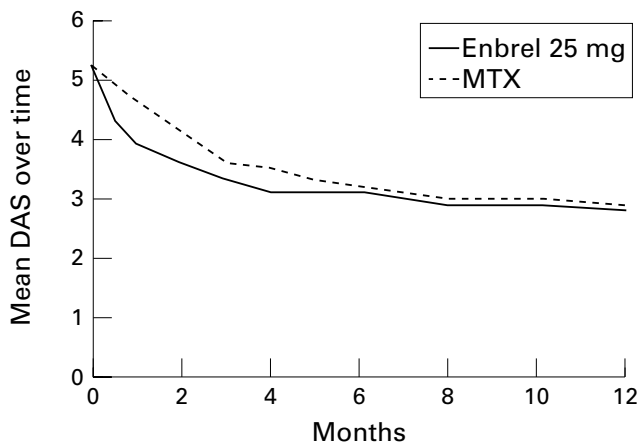

Figure 1 The change from baseline over time in the Disease Activity Score (DAS). Improvement in the etanercept $25 \mathrm{mg}$ group was more rapid than the methotrexate group.

\section{ETANERCEPT IN METHOTREXATE NAIVE RA} PATIENTS

With demonstrated evidence that etanercept controls the signs and symptoms in patients with longstanding RA, a study was initiated to determine the ability of etanercept to improve signs and symptoms and to slow radiographic progression in RA patients who had not been treated previously with methotrexate. ${ }^{18} 19$ This multicentre, randomised, double blind study compared improvement in signs and symptoms and radiographic progression in RA patients treated with etanercept with those treated with high dose oral methotrexate, which has been considered to be the most effective single agent DMARD available to treat RA. The trial was conducted in 632 patients who had active RA of three years or less duration, who had never been treated with methotrexate, and who had risk factors for rapidly progressive joint damage (that is, were rheumatoid factor positive or had erosions on baseline radiographs). Patients received either $25 \mathrm{mg}$ or $10 \mathrm{mg}$ of etanercept subcutaneously twice a week or methotrexate, rapidly dose escalated from $7.5 \mathrm{mg}$ to $20 \mathrm{mg}$ orally per week over an eight week period. End points included the ability of etanercept, compared with methotrexate, to reduce the rate of joint damage over 12 months and to reduce the signs and symptoms of RA over six months.

Although mean duration of disease of patients at study entry was less than one year, patients had very active disease, with baseline

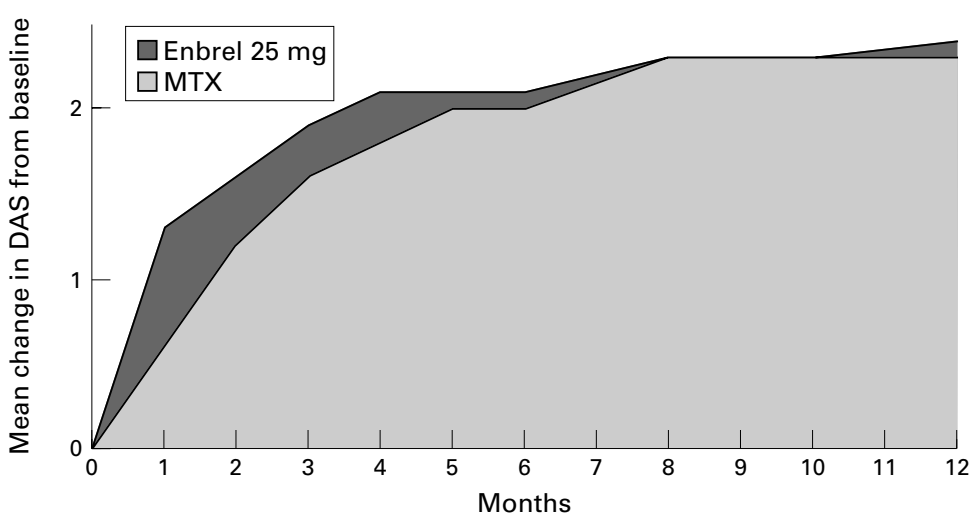

Figure 2 The area under the curve (AUC) of the change from baseline in the Disease Activity Score (DAS). Improvement in the etanercept $25 \mathrm{mg}$ group was significantly better than the methotrexate group over both the six and 12 month time periods $(p=0.002$ and $p=0.031$, respectively). means of 24 swollen joints and 31 tender joints and a baseline mean Health Assessment Questionnaire (HAQ) score of 1.4. Eighty eight per cent of patients were positive for rheumatoid factor. The mean disease activity score (DAS) for all patients in the trial was 5.2, with 90\% having high disease activity (DAS > 3.7) at baseline. ${ }^{20} 21$

Results of the trial showed that etanercept slowed disease progression and improved signs and symptoms of disease activity. As in previous trials, the etanercept $25 \mathrm{mg}$ twice weekly dose was found to be more effective than the etanercept $10 \mathrm{mg}$ twice weekly dose. More patients in the etanercept $25 \mathrm{mg}$ group than in the MTX group completed one year of study drug compared with methotrexate $(85 \%$ versus $79 \%$, respectively).

Improvement in the etanercept $25 \mathrm{mg}$ group was seen early in the study, as shown by improvement in the DAS (fig 1). At one month, the mean DAS fell to 3.9 for the etanercept 25 mg group, compared with 4.6 for the methotrexate group $(\mathrm{p}<0.001)$. At two months, the mean DAS was 3.6 for the etanercept 25 group compared with 4.1 for the methotrexate group $(\mathrm{p}=0.026)$. At six months, the mean DAS for the etanercept $25 \mathrm{mg}$ group was 3.2 compared with 3.1 for the methotrexate group $(p=N S)$.

Response as measured by the European League Against Rheumatism (EULAR) criteria was also seen early in the study. ${ }^{21}$ At one month, $15 \%$ of the etanercept 25 group had achieved a "good" response and an additional $44 \%$ a "moderate" response, compared with $3 \%$ and $27 \%$, respectively, in the methotrexate group. At two months, $18 \%$ of the etanercept 25 group had achieved a "good" response and an additional $54 \%$ a "moderate" response, compared with $7 \%$ and $47 \%$, respectively, in the methotrexate group. At six months, $32 \%$ of the etanercept 25 group had achieved a "good" response and an additional $48 \%$ a "moderate" response. In comparison, $31 \%$ of the methotrexate group had achieved a "good" response and an additional $44 \%$ a "moderate" response at six months.

To determine the cumulative response over time, these results were quantified as the area under the curve (AUC) of the improvement in DAS. ${ }^{192}$ This time integrated improvement was significantly better in the etanercept $25 \mathrm{mg}$ group compared with methotrexate at six months $(0.9 v 0.7$, respectively, $\mathrm{p}=0.002)$ and at 12 months (1.9 v 1.7, respectively, $\mathrm{p}=0.031$; fig 2).

Both etanercept $25 \mathrm{mg}$ and methotrexate were shown to be effective DMARDs with respect to slowing the rate of radiographic progression. At baseline, the predicted annual rate of progression (baseline Sharp score divided by duration of disease) was 9.1 Sharp units, with no significant differences among the treatment groups. At one year, $60 \%$ of methotrexate patients had no radiographic progression of erosions compared with $72 \%$ of etanercept $25 \mathrm{mg}$ patients $(\mathrm{p}=0.007)$. The rates of progression between methotrexate and etanercept $25 \mathrm{mg}$ were significantly different with respect to change in erosions $(p<0.001)$ and 


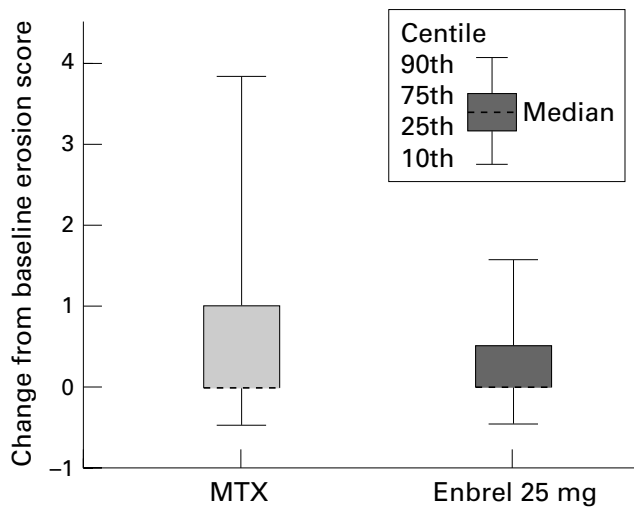

Figure 3 Box plot showing change from baseline in erosion score at one year for patients receiving etanercept $25 \mathrm{mg}$ and methotrexate. The median change for each was 0 . The difference between the etanercept $25 \mathrm{mg}$ and the methotrexate groups was statistically significant $(p=$ 0.002).

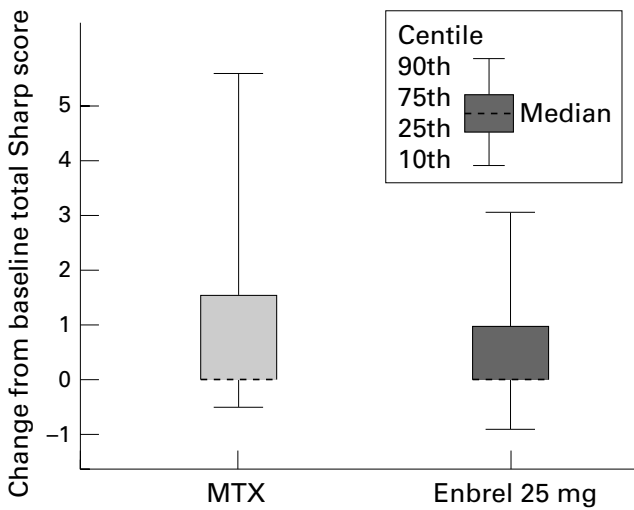

Figure 4 Box plot showing change from baseline in Total Sharp Score at one year for patients receiving etanercept and methotrexate. The median change for each was 0 . The difference between the etanercept $25 \mathrm{mg}$ and the methotrexate groups did not meet statistical significance ( $p$ $=0.110$ ).

approached significance with respect to change in Sharp score $(\mathrm{p}=0.110)$.

At one year, the median changes in joint erosion, joint space narrowing, and total Sharp score were zero for both groups. Box plots showing changes in erosions and total Sharp score at one year are shown in figures 3 and 4 . The range of increase was smaller in the etanercept $25 \mathrm{mg}$ group than in the methotrexate group. In $90 \%$ of patients in the etanercept 25 group, the change in erosion score at 12 months was $\leqslant 2$ points, compared with the methotrexate group where, in $90 \%$ of patients, the change in erosion score was $\leqslant 4$ points.

More patients withdrew from the study for adverse events in the methotrexate group $(11 \%)$ than in either etanercept group $(6 \%$ in the $10 \mathrm{mg}$ and $5 \%$ in the $25 \mathrm{mg}$ group). The safety profile of etanercept in this study was similar to that observed in earlier studies. Etanercept was well tolerated and the most commonly reported events in patients receiving etanercept were mild to moderate injection site reactions that did not complicate treatment. Adverse events that occurred at a higher rate in patients receiving methotrexate compared with those receiving etanercept included nausea, rash, mouth ulcers, alopecia, vomiting, and epistaxis. Pneumonitis, seen only in the meth- otrexate group, developed in three patients. The frequency of any infection was higher in the methotrexate group than the etanercept 25 mg group $(p<0.01)$, but the rates of infections requiring intravenous antibiotics or hospitalisation were comparable in all three treatment arms.

In summary, etanercept provided rapid and effective improvement in measures of disease activity in patients with active, early RA and was significantly more effective than methotrexate in reducing the rate of joint erosions. Etanercept was generally well tolerated and, when compared with methotrexate in this population, was associated with fewer adverse events and withdrawals.

\section{ETANERCEPT IN PSORIATIC ARTHRITIS}

The efficacy of etanercept was also studied in a placebo controlled, double blind study of 60 patients with severe psoriatic arthritis (PsA) who were treated with either etanercept $25 \mathrm{mg}$ or placebo subcutaneously twice weekly for 12 weeks. ${ }^{23}$ The end points of the trial were improvement in the PsA response criteria (PsARC) as well as the ACR20 response. The effect of etanercept on dermatological response was analysed in a subset of patients who had $\geqslant$ $3 \%$ of total body involvement of their psoriasis by assessing target lesions and by using the Psoriasis Area and Severity Index (PASI), a composite measure based on scale, erythema, and induration of psoriasis lesions.

Patients treated with etanercept had significant improvement in disease activity compared with those given placebo. At 12 weeks, $87 \%$ of patients treated with etanercept met the PsARC criteria compared with $23 \%$ of patients treated with placebo $(\mathrm{p}<0.001)$. Seventy three per cent of etanercept treated patients achieved an ACR 20 response compared with $13 \%$ of placebo treated patients $(p<0.001)$. Some patients treated with etanercept had a $100 \%$ improvement in disease activity, including four with no tender joints, seven with no swollen joints, 11 with no morning stiffness, and 11 with a HAQ zero disability score. Of the 38 patients evaluable for psoriasis, patients receiving etanercept experienced a median improvement in the PASI score of $46 \%$ compared with $9 \%$ in the placebo group $(p=0.003)$. No deaths, serious adverse events, or serious infections occurred in either treatment arm.

FURTHER INDICATIONS FOR ETANERCEPT

TNF is an important inflammatory disease mediator in a wide spectrum of articular diseases, including RA, JRA, and PsA. Etanercept is an effective inhibitor of TNF, has been shown to provide rapid and sustained improvement in these diseases, and is an important new treatment option for patients with these conditions. Etanercept is currently being studied in other disorders in which TNF seems to play an important pathogenic part, including chronic heart failure, sarcoidosis, ankylosing spondylitis, and Wegener's granulomatosis. 
1 Garrison L, McDonnell ND. Etanercept: therapeutic use in patients with rheumatoid arthritis. Ann Rheum Dis patients with rheumatoid
$1999 ; 58$ (suppl 1):165-9.

2 Bertolini DR, Nedwin GE, Bringman TS, et al. Stimulation of bone resorption and inhibition of bone formation in vitro by human tumour necrosis factors. Nature 1986;319:51618.

3 Dayer J-M, Beutler B, Cerami A. Cachectin/tumor necrosis factor stimulates collagenase and prostaglandin $\mathrm{E} 2$ production by human synovial cells and dermal fibroblasts. J Exp Med 1985;162:2163-8.

4 Lader CS, Flanagan AM. Prostaglandin E2, interleukin $1 \alpha$, and tumor necrosis factor- $\alpha$ increase human osteoclast formation and bone resorption in vitro. Endocrinology 1998; 139:3157-64.

5 Nawroth PP, Bank I, Handley D, et al. Tumor necrosis factor/cachectin interacts with endothelial cell receptors to induce release of interleukin 1. J Exp Med 1986;163:136375 .

6 Butler DM, Maini RN, Feldmann M, et al. Modulation of proinflammatory cytokine release in rheumatoid synovial membrane cell cultures. Comparison of monoclonal ant TNF-alpha antibody with the interleukin-1 receptor antagonist. Eur Cytokine Netw 1995;6:225-30

7 Brennan FM, Chantry D, Jackson A, et al. Inhibitory effect of TNF $\alpha$ antibodies on synovial cell interleukin-1 production in rheumatoid arthritis. Lancet 1989;ii:244-7.

8 Mohler KM, Torrance DS, Smith CA, et al. Soluble tumor necrosis factor (TNF) receptors are effective therapeutic agents in lethal endotoxemia and function simultaneously as both TNF carriers and TNF antagonists. J Immuno 1993;151:1548-61.

9 Barone D, Krantz C, Lambert D, et al. Comparative analysis of the ability of etanercept and infliximab to lyse TNF-expressing cells in a complement dependent fashion. Arthritis Rheum 1999;42:S369.

10 Wooley PH, Dutcher J, Widmer MB, et al. Influence of a recombinant human soluble tumor necrosis factor receptor Fc fusion protein on type II collagen-induced arthritis in Fc fusion protein on type II collagen
mice. J Immunol 1993;151:6602-7.

11 Moreland LW, Margolies G, Heck LW, et al. Recombinant soluble tumor necrosis factor receptor (p80) fusion protein: toxicity and dose finding trial in refractory rheumatoid arthritis. J Rheumatol 1996;23:1849-55.
12 Moreland LW, Baumgartner SW, Schiff MH, et al. Treatment of rheumatoid arthritis with a recombinant human tumor necrosis factor receptor (p75)-Fc fusion protein. N Engl J Med 1997;337:141-7.

13 Moreland LW, Schiff MH, Baumgartner SW, et al. Etanercept therapy in rheumatoid arthritis. A randomized, controlled trial. Ann Intern Med 1999;130:478-86.

14 Weinblatt ME, Kremer JM, Bankhurst AD, et al. A trial of etanercept, a recombinant tumor necrosis factor receptor:Fc fusion protein, in patients with rheumatoid arthritis receiving methotrexate. N Engl J Med 1999;340: 253-9.

15 Lovell DJ, Giannini EH, Reiff A, et al. Etanercept in children with polyarticular juvenile rheumatoid arthritis. $\mathrm{N}$ Engl J Med 2000;342:763-9.

16 Moreland LM, Cohen SB, Baumgartner S, et al. Long-term use of Enbrel in patients with DMARD-refractory rheumatoid arthritis. Arthritis Rheum 1999:42:S401.

17 Parker SL, Tong T, Bolder S, et al. Cancer statistics, 1997. CA Cancer J Clin 1997:47:5-27.

18 Finck B, Martin R, Fleischmann R, et al. A Phase III trial of etanercept vs methotrexate (MTX) in early rheumatoid arthritis (Enbrel ERA trial). Arthritis Rheum 1999;42: S117.

19 Schiff M, Weaver A, Keystone E, et al. Comparison of ACR response, numeric ACR, and ACR AUC as measures of clinical improvement in RA clinical trials. Arthritis Rheum 1999;42:S81.

20 van der Heijde DMFM, van't Hoff MA, van Riel PLCM, et $a l$. Judging disease activity in clinical practice in rheumatoid arthritis: First step in the development of a disease activity score. Ann Rheum Dis 1990;49:916-20.

21 van Gestel AM, Prevoo MLL, van't Hof MA, et al. Development and validation of the European League Against Rheumatism response criteria for rheumatoid arthritis. Arthritis Rheum 1996;39:34-40.

22 Pham B, Cranney A, Boers M, et al. Validity of area-underthe-curve analysis to summarize effect in rheumatoid arthritis clinical trials. J Rheumatol 1999;26:712-16.

23 Mease PJ, Goffe BS, Metz J, et al. Randomized trial of etanercent (Enbrel) in the treatment of psoriatic arthritis and psoriasis. Lancet 2000;356:385-90. 